\title{
Self-assembly around curved surfaces
}

\author{
Luke A. Stewart ${ }^{1,2,3}$, Graham D. Marshal ${ }^{1,2,3}$, Judith M. Dawes ${ }^{1,2,3}$, Michael J. Withford ${ }^{1,2,3}$ \\ and Adel Rahmani ${ }^{1,4}$
}

1. Centre for Ultrahigh-bandwidth Devices for Optical Systems (CUDOS),

2. MQ Photonics research centre,

3. Department of Physics, Macquarie University

North Ryde, NSW 2109, Australia.

4. Department of Mathematical Sciences, University of Technology

Broadway, NSW 2007, Australia

Fax : +61298508115 ,

E-Mail: 1stew@ics.mq.edu.au

\begin{abstract}
We present a parametric study of self-assembled photonic crystal growth as a function of radius of curvature. To do this, we used a combination of microscope slides, glass capillaries and optical fibres as substrates to grow the self-assembled films on. Microscope and SEM images, as well as broadband transmission spectra were used to characterise the crystal, and the effect the changing surface curvature had on the crystal quality. Limitations for fabricating the crystals on highly curved surfaces will be presented.
\end{abstract}

KEYWORDS: Photonic crystal, photonic, crystail, opal, seif assembiy, fibre, fuber.

Photonics: Design, Technology, and Packaging III

edited by Wieslaw Z. Krolikowski, Costas M. Soukoulis, Ping Koy Lam, Timothy J. Davis, Shanhui Fan, Yuri S. Kivshar Proc. of SPIE Vol. 6801, 68011A, (2008) - 0277-786/108/\$18 - doi: 10.1117/12.769338

Proc. of SPIE Vol. $680168011 \mathrm{~A}-1$ 


\section{INTRODUCTION}

Photonic crystals are structures with periodic features on scales comparable to the wavelength of light. That is hundreds of nanometres to a several micrometres. Photonic crystals interact with light in well defined ways. Namely, they have the ability to block specific wavelengths of light from existing within them. This is analogous to the control that semiconductor materiais exercise over electrons, which paved the way to the electronic processing chip

Self-assembly is a promising technique for fabricating three-dimensional photonic crystals due to the relative simplicity of the fabrication process and the ability of the finished structure to possess a full photonic bandgap [1]. Self-assembly involves aking a sample of uniformly sized microspheres and providing conditions in which they will arrange themselves periodically. Self-assembled photonic crystals are often referred to as opals, since they share similar iridescence and microscopic structure. An example of these properties is shown below in Figure 1.

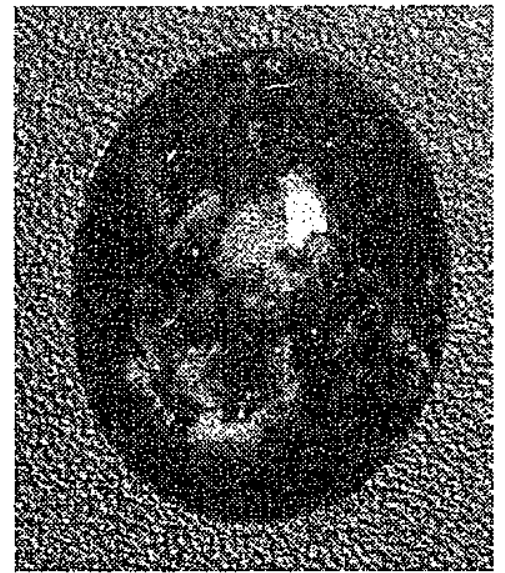

(a)

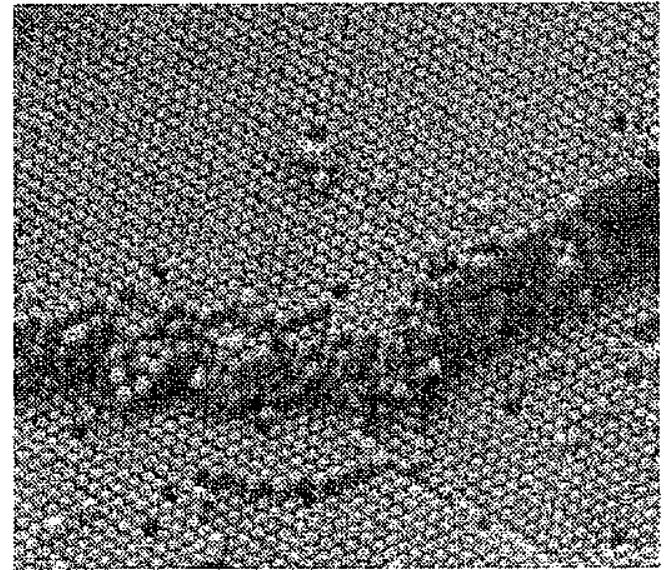

(b)

Figure 1: (a) An opal gemstone, displaying its brilliant iridescence [2]; (b) An example of a high magnification image of an opal, showing a periodic arrangement of tiny spheres

Recent advances in fabricating selfwassembled photonic crystals with excellent long range order [3] has led to possible applications in sensors, switchable displays and even decorative applications [4-6]. Kitaev ef al. have also shown that self-assembled photonic crystals can be grown around curved surfaces [7]. They were able to grow an opal structure around a $125 \mu \mathrm{m}$ silica optical fibre and show a uniform bandgap at all radial angles with respect to the core. This was proposed as being a precursor for a new type photonic bandgap fibre, since the voids in the opal could subsecuently be fllled with silicon and the spheres and fibre removed by chemical etching. The problem with this is that it leaves a core with a $125 \mathrm{fm}$ dianeter, which is simply too targe for most fibre applications.

In this study, we examine the effects of growing opals around curved surfaces of various diameters. In particular, we look at the macroscopic and microscopic crystal quality as the diameter is reduced. The motivation of this study is to gain insights into problems that may arise in making these new photonic bandgap fibres with a more usefil. core diameter.

\section{EXPTRIMENTAL METHODS}

Microscope slides $(75 \times 25 \times 1 \mathrm{~mm}$, Fisher) were cut in half along the long sides and used as substrates. The substrates along with scintillation vials (20mL, Fisher) were soaked in a Piranha solution $\left(\mathrm{H}_{2} \mathrm{SO}_{4}: \mathrm{H}_{2} \mathrm{O}_{2}, 3: 1\right)$ for 10 minutes. They were rinsed with ultra-pure water directly from a Milli-Q water system (18.2 M $\Omega \mathrm{cm}-1$ ), and then in absolute ethanol (Univas - analytical reagerit) before being dried in a stream of nitrogen. The cleaning process leaves the substrates 
hydrophilic, which allows for uniform growth. For studies of growth on curved surfaces, glass capillaries and optical fibres were used as substrates, but the methods remained the same. The optical fibre (Coning SMF-28) was stripped of is jacket before use.

The substrates were placed close to vertical in a scintillation vial, containing $10 \mathrm{~mL}$ of colloidal solution. The colloidal solution consisted of polystyrene microspheres (Bangs Laboratories, $220 \mathrm{~nm}$ diameter, $10 \%$ wt) diluted by ultra-pure water to a dilution of $0.2 \%$ weight of polystyrene. The vial was placed inside a temperature controlled oven, set to $60^{\circ} \mathrm{C}$. This purgose of the heating was to speed up the evaporation process, as well as to give the microspheres enough thermal energy to stop sedimentation. The experiment was left for approximately 24 hours to let the solution evaporate. A schematic of the growth method is illustrated below in Figure 2.

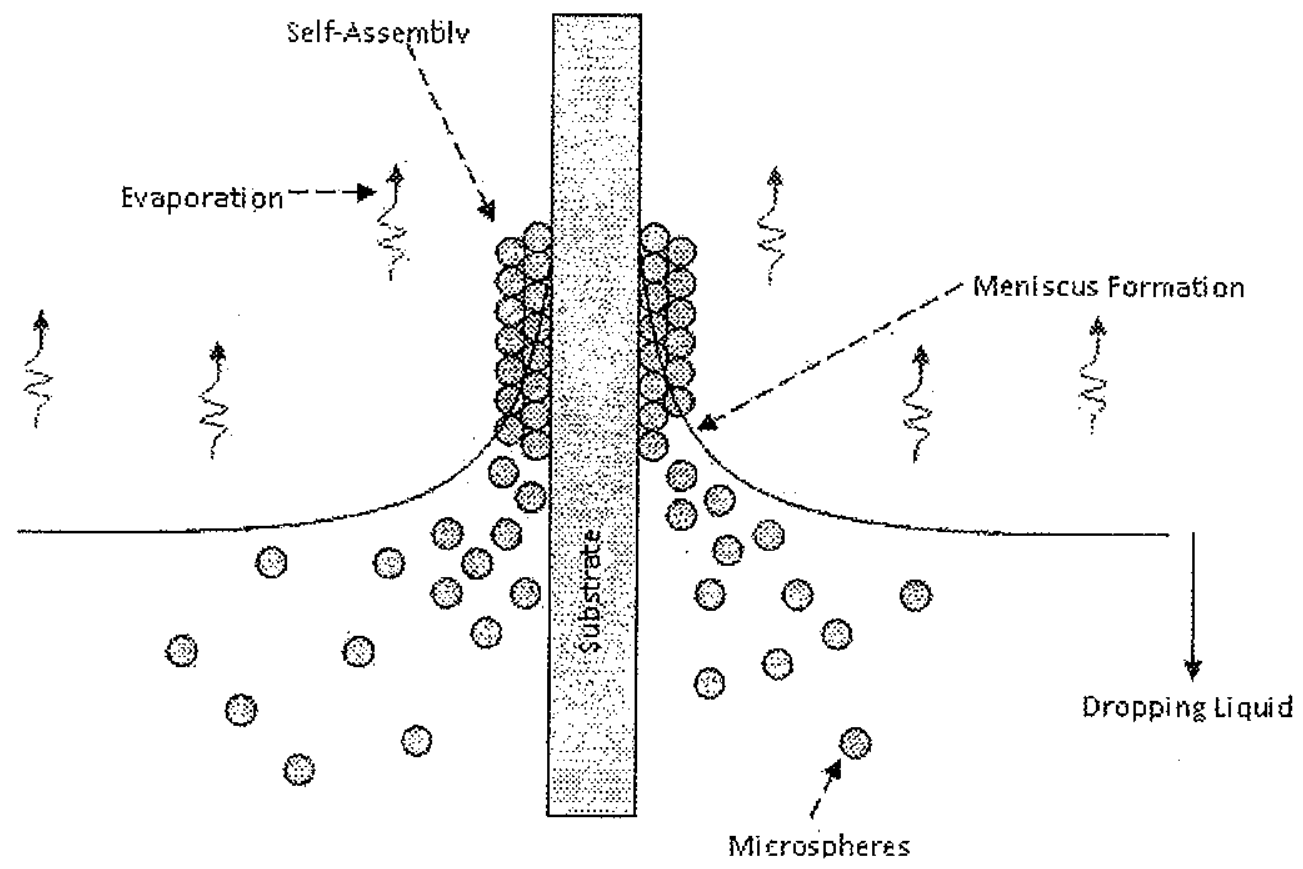

Figure 2: Schematic representation of the vertical deposition method.

The method uses a near vertical substrate immersed in a solution of the polystyrene or silica microspheres. As the solvent evaporates, the meniscus draws down the edge of the substrate, forcing the spheres to be deposited. Nuturully, the spheres try to arrange themselves in such a way that they have the lowest potential energy. This arrangement happens to be a face-centred cubic lattice, although other anangements are sometimes observed.

Characterisation of the opal film was initially performed by an Olympus differential interference contrast microscope in reflection mode. This allowed for examination of large scale cracking in the samples, as well as viewing any opalescence. A scanning electron microscope (SEM) (Jeol JSM-6490LV) was used to characterise the microscopic siructure of the opal films and determine if the structures were periodic. A Cary 5E UV-Vis-NIR Dual-Beam Spectrophotometer was used to take spectra of transmission and reflection of the opal films. A tungsten-halogen light source (ocean optics) in conjunction with an Ocean Optics UV-VIS spectrometer was used to taken spectral measurements through the opals clad fibres. 


\section{RESULTS}

The image shown below in Figure 3 is a microscope image of an opal tilm. The green colour that is displayed is an indication of the position of the plotonic bandgap in the spectrum, since it is reflecting this colour back to the eyepiece from a white light illumination. The position of this bandgap is determined by the size of the spheres, and their refractive index. This image also displays a large amount of cracking, which is a function of the film shrinking as it dries out.

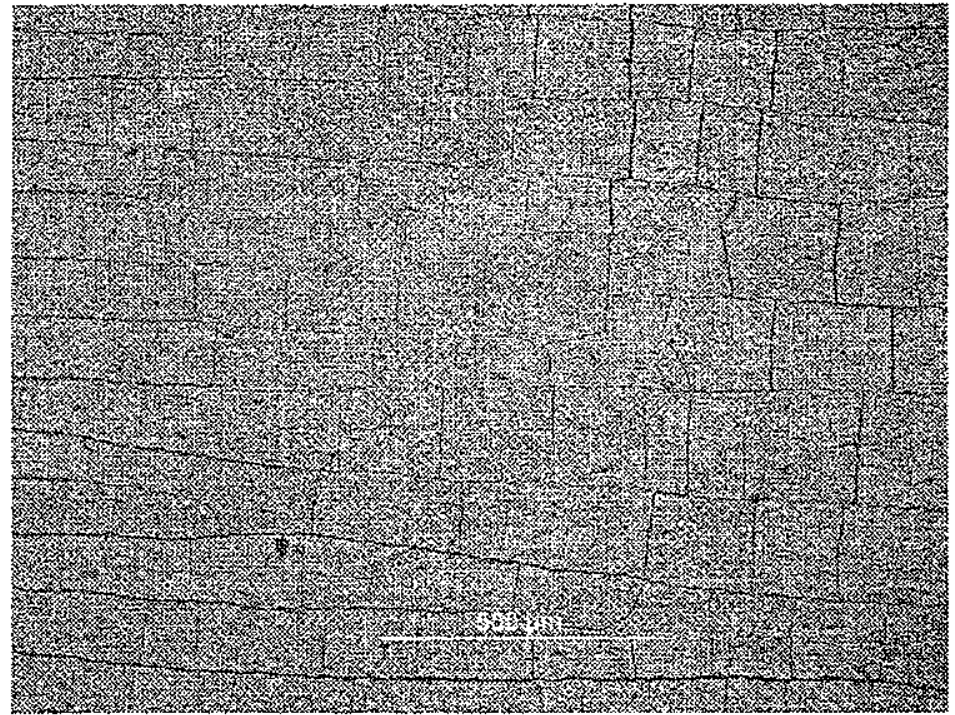

Figure 3: Microscope image of an opal film.

A typical SEM of a fabricated opal is shown below in Figure 4. Figure 4(a) is an image of the top layer of a film, showing that the spheres arrange themselves in a hexagonal lattice in each layer. There are some slight dislocations in the film, as well as some missing spheres, but generally the ordering is very periodic. Figure $4(b)$ shows a cross section of the fim, indicating how the layers stack. Analysis of such images show that the films are predominantly stacked in a face-centred cubic arrangement.

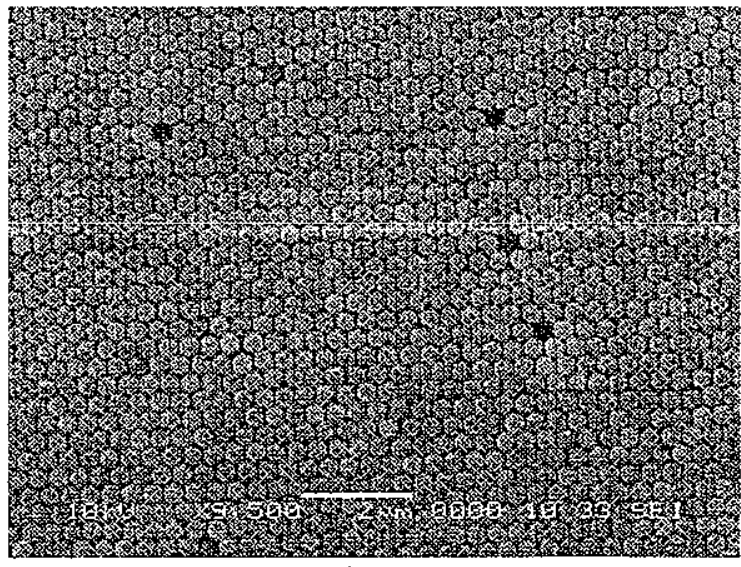

(a)

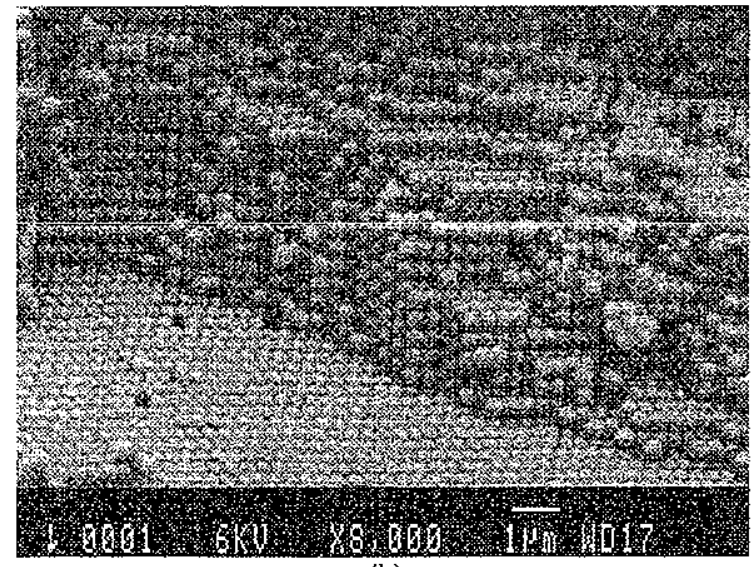

(b)

Figure 4: SEM images of an opal fabricated using the vertical deposition method. (a) Shows the top layer with its hexagonal lattice, and (b) shows the 3D face-centred cubic lattice. 
Figure 5 (below) shows a typical transmission spectrum of an opal film. In this case, the spectrum shows a sharp dip in transmission around $520 \mathrm{~mm}$. This indicates that the opal has a photonic bandgap that is centred at this wavelength (for normal incidence). The FWHM of this bandgap is approximately $50 \mathrm{~nm}$.

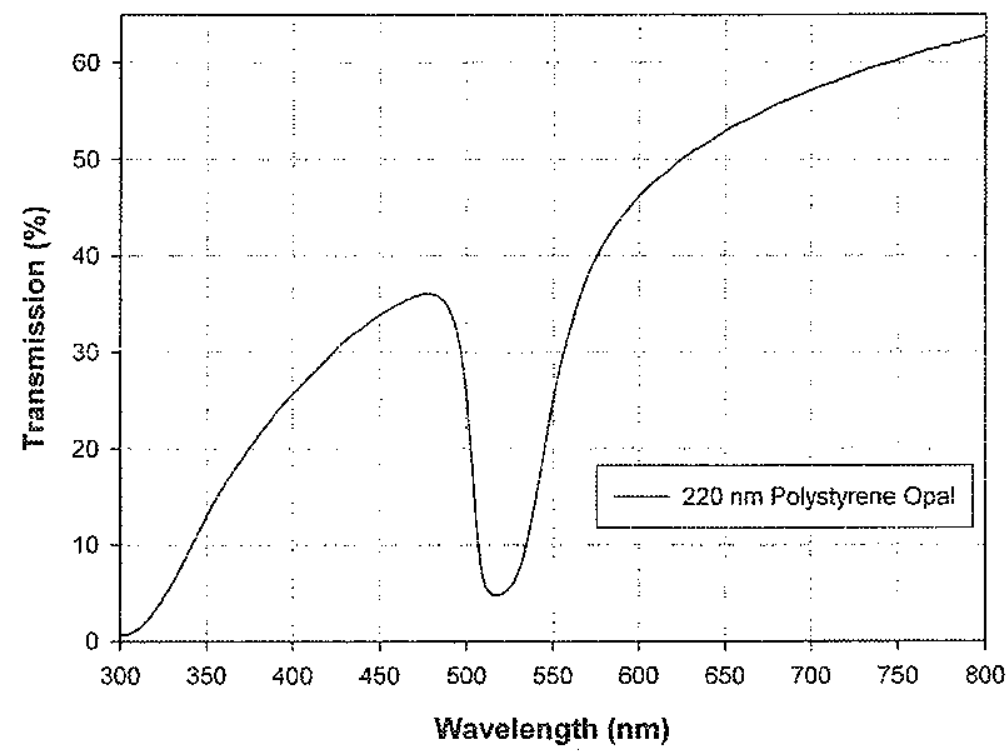

Figure 5: Transmission spectrum of an opal film made from $220 \mathrm{~nm}$ polystyrene microspheres. The spectrum indicates a photonic bandgap centred at a wavelength of $520 \mathrm{~nm}$.

The position of this bandgap, $\lambda_{B}$, is governed by the following equation, which is a combination of Bragg's Law and Snell's Law [8]:

$$
\lambda_{B}=2 D \sqrt{\frac{2}{3}\left(n_{e f f}^{2}-\sin ^{2} \theta\right)}
$$

where

$$
\begin{aligned}
& D=\text { sphere diameter } \\
& n_{t / f}=\text { effective refiactive index } \\
& \theta=\text { angle of incidence }
\end{aligned}
$$

The effective refractive index is govened by the material of the spheres being used and the way the pack together. Sincc the spheres tend to pack in a face-centred cubic lattice, for a given sphere material the position of the bandgap depends only on the size of the spheres $(D)$ and the angle of incidence $(\theta)$. Figure 6 (below) shows plots of the effect of $D$ and $\theta$ on the position of the bandgap, for both theoretical and measured data. The graph in tigure 6(a) shows a linear trend between bandgap position and sphere diameter, and shows that the experimental data follows the trend closely. The measured data for the effect of angle of incidence also matches the predicted trend well. Small angles $\left(<20^{\circ}\right)$ prove to have little effect on the position of the bandgap, but beyond this the curve steepens and the angle becomes more critical. 

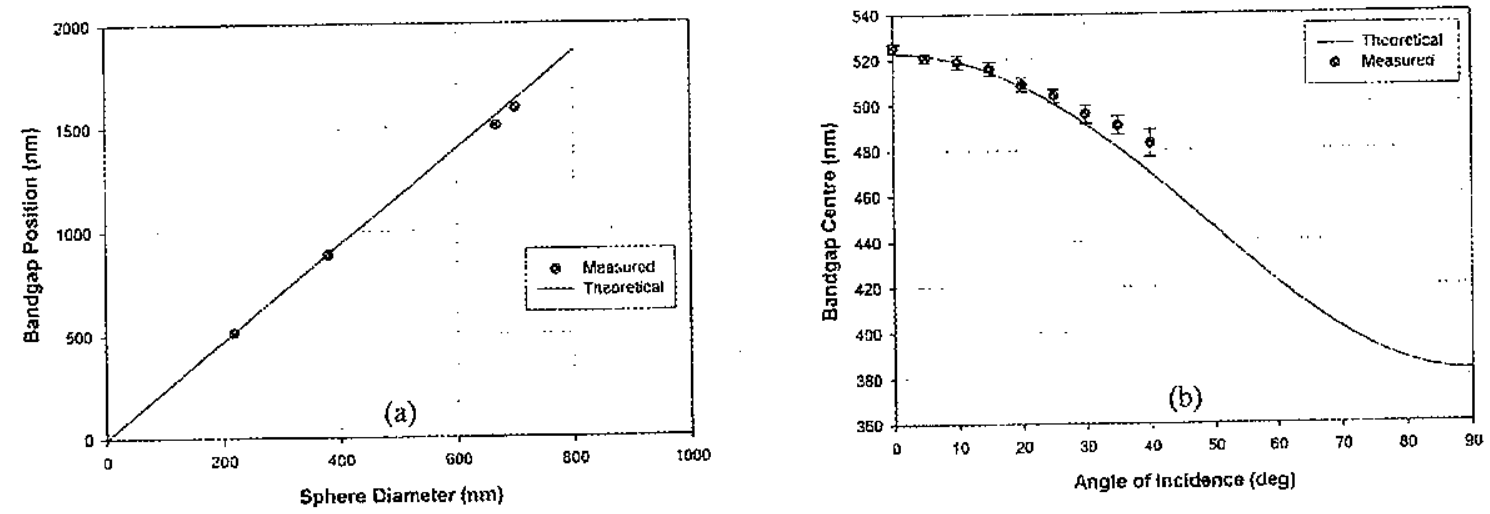

Figure 6: (a) Effect of sphere diameter, $D$, and (b) angle of incidence, $\theta$, on the position of the bandgap

The schematic shown below in Figure 7 shows the expected effect that a curved substrate would have on the growth of an opal fim. In the extreme case of a flat substrate, the spheres can theoretically order themselves perfectly into a facecentred cubic lattice (since this is the lowest energy that they can exist). As we move to a substrate with slightly more curvature, we see that the spheres can still arrange themselves, but as we add more layers, adjacent spheres become further apart. When we look at the case of a high radius of curvature, there is little periodicity in the structure.

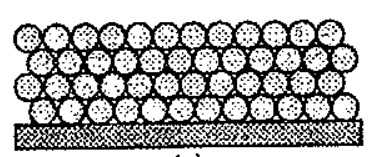

(a)

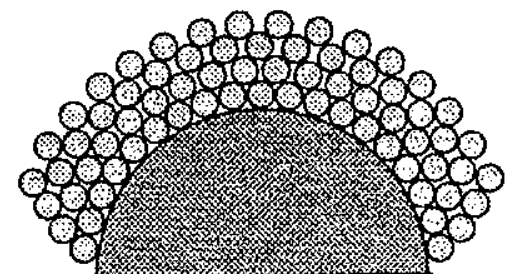

(b)

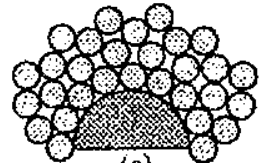

(c)

Figurc 7: Schematic of nicrosphere ordering in substrates with various radii of curvature. As the curvature increases (i.e. the substrate radius becomes smaller) the spheres cannot retain a true face-centred cubic stacking.

To investigate the effect of growing opals on curved surfaces, we used a variety of different substrates. The control sample was a microscope slide, which was the extreme case of an infinite radius of curvature. Giass capiliaries were used as an intermediate step, having an ouler diameter of around $1 \mathrm{~mm}$. Smaller radii were achieved by using standard single mode optical fibres (diameter of $125 \mu \mathrm{m}$ ).

Figure 8 below compares microscope images of an opal grown on a flat glass substrate, a glass capillary and on an optical fibre. Quantitatively, cracks seem to appear on a average every 100-200 microns on the flat sample, every 80-100 microns on the capillary and every 30-50 microns on the optical fibre. There is a definite trend between the curvalure of the surface and the macroscopic cracking in the opal grown on it. The higher curvature surfaces tend to have more cracks per unit area. 


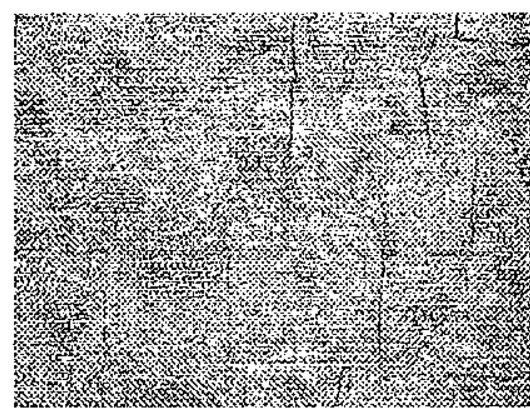

(a)

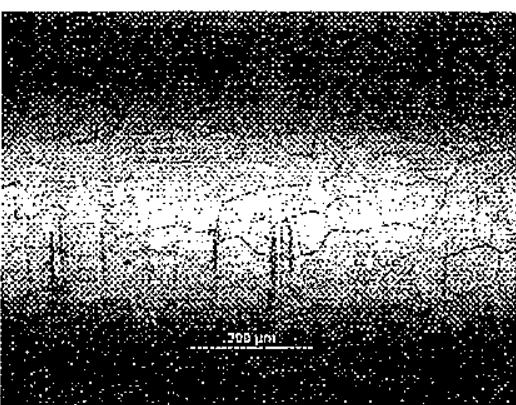

(b)

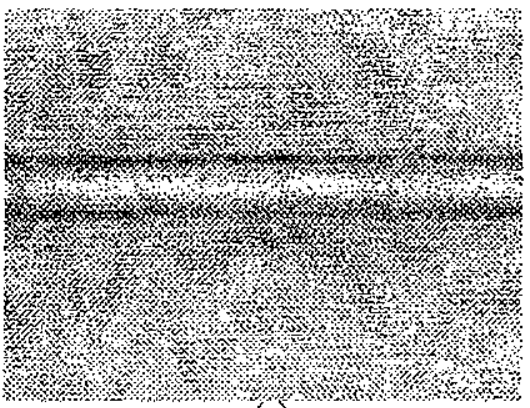

(c)

Figure 8: Difference between an opal grown on (a) a flat glass substrate (b) a glass capillary and (c) a $125 \mu$ m diameter optical fibre. Notice that there is far more cracking per unit area in the opal that is grown on the higher curvature surfaces.

High magnification images from a SEM provide the greatest insight into the effect of the substrate curvature on the structure of the opal. Fjgure 9 (below) shows SEM images of an opal grown on a flat substrate (a), a glass capillary (b) and an optical fibre (c). It is obvious that the ordering of the spheres on the flat substrate is the most periodic and has the least dislocations in the lattice. The opal grown on the capillary still has decent ordering, but there are many more lattice dislocations. The opal grown on the optical fibre has the worst ordering. Although the spheres are still packed closely together, they are not ordered as perfectly as the flatter substrates. This clearly shows that the curvature of the substrate has a big impact on the ordering of the opal.

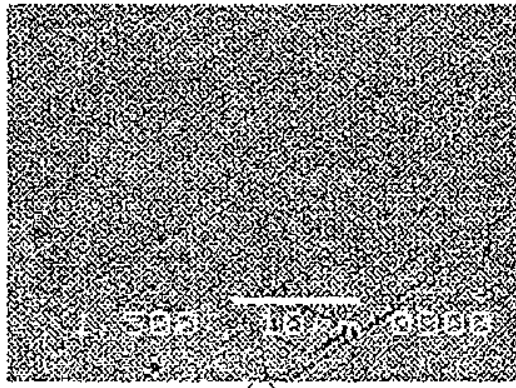

(a)

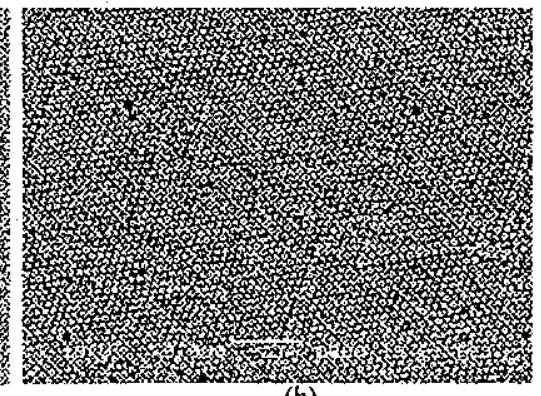

(b)

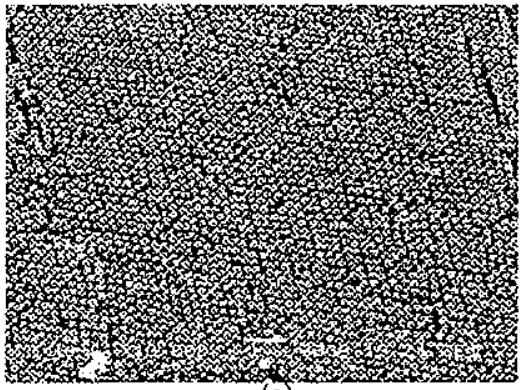

(c)

Figure 9: SEM images of opals grown on various surfaces: (a) flat surface, (b) capillary and (c) optical fibre. It is obvious that as the substrate curvature increases, the periodicity of the top layer of spheres gets worse.

Even though the periodicity of the opals grown on curved surfaces is not perfect, they can still display a photonic bandgap in their transmission spectra. This is becanse the inter-plane distance remains fairly constant. Figure 10 (below) shows the transmission spectra through an opat elau optical fibre, at various angles of inciüence. The transinission spectra was taken by coupling a tungsten halogen light source into a multimode optical fibre, and butt coupling this to another multimode fibre which was connected to a detector. The fibres were then separated to allow for the opal clad fibre to be placed between them, tilted by $90^{\circ}$. This setup is shown in Figure $10(a)$ below).

A photonic bandgap can be seen centred at a wavelength of $520 \mathrm{~nm}$, the same position as for the opal grown on the flat substrate (figure 5). Notice that compared to the opal grown on the flat surface, this bandgap is not as deep, and appears to be broader. The FWHM is approximately $100 \mathrm{~nm}$ compared to $50 \mathrm{~nm}$ in the standard opal. This difference comes about because the periodicity is not as good. Notice also that the iransmission spectrum is now independent of the angle of rotation of the fibre. This occurs because we now have a circularly symmetric crystal, which contrasts the strong angular dependence of the bandgap in the opal grown on a flat surface. 

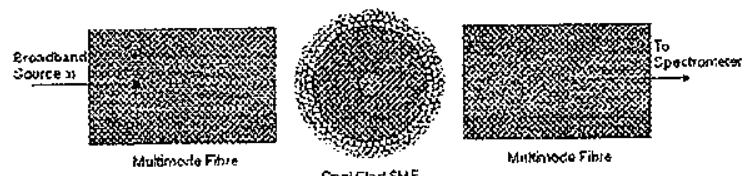

(a)

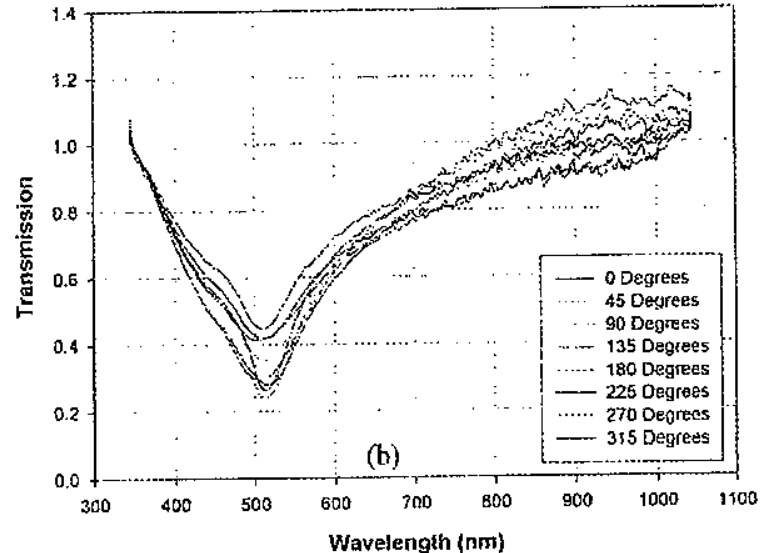

elengith (nm)

Figure 10: (a) Experimental setup for probing an opal clad optical fibre, and (b) the corresponding transmission spectra.

\section{CONCLUSIONS:}

Self-assembly is a simple and effective techique for fabricating large area photonic crystals. Standard self-assembled photonic crystals, or opals, are made up of a face-centred cubic arrangement of microspheres. The top layer of these opals (parallel to the substrate) is arranged in a hexagonal lattice. Spectrally, these crystals display a deep photonic bandgap, with a FWHM of approximately $50 \mathrm{~nm}$. The position of this bandgap is strongly dependent on the size of the spheres and the angle of incidence.

When the substrate is curved, the spheres cannot assemble themselves as perfectly as they would on a flat substrate. This leads to an increase in macroscopic cracking throughout the sample, and also more dislocations at a microscopic level. This means that when the sample is probed in transmission, the photonic bandgap broadens. Using a 125 micron diameter fibre as a substrate, the FWHM broadened to $100 \mathrm{~nm}$ - double that of the flat substrate.

The effects seen in this study will provide insights into what will be expected when trying to scale these 'photonic bandgap fibres' down, such that the core diameter is a more useful size. The issues of macroscopic cracking may be addressable, through moditied growth techniques and better drying techniques. For example Leyrer ef al [9] have shown that by adding a soft polymer coating to the microspheres, crack free areas of up to $2.6 \times 2.6 \mathrm{~cm}$ can be achieved. The sof polymer actually fills the voids in the opal as it dries, giving it some elasticity and stopping the cracks from occurring. The drawback of this is the loss of refractive index contrast.

The micrescopic defects may cause more problems. Substrates with high curvature cannot support many layers of spheres with perfect packing. The only way to lessen the effect at high curvatures is to use smaller spheres, but this in curn changes the position of the photonic bandgap. There is also a trade-off between the radius of the substrate, and the FWHM of the photonic bandgap. All these factors must be considered if scaling down to these smaller radii is to be realised.

\section{ACKNOWLEDGMENTS}

This work was produced with the assistance of the Australian Research Council under the ARC Centres of Excellence and LIEF programs. 


\section{REFERENCES}

1. K. Busch and S. John, Photonic band gap formation in certain self-organizing systems, Physical Review E 58(3), p. 3896-3908 (1998).

2. hilp://www.profoundglass.com/

3. S. Wong et al, Colloidal Crystal Films: Advances in Universality and Perfection, J. AM. CHEM. SOC. 125(50), p15589-15598 (2003).

4. A. Arsenault tet al, From colour fingerprinting to the control of photoluminescence in elastic photonic crystals, Nature Materials 5, p179-184 (2006)

5. A. Arsenault et al, Photonic-crystal fill-colour displays, Nature Photonics 1, p468-472 (2007)

6. O. Pursiainen et al, Nanoparticle -t2med structural color from polymer opals, Optics Express 15(15), p95539561 (2007).

7. V. Kitaev et al, Colloidal pholonic crystal cladded optical fibers: Towards a new type of photonic band gap fiber, Optics Express 13(17), p. 6454-6459 (2005).

8. J. Huang et a\}, Anomalous Coherent Backscattering of Light from Opal Photonic Crystals, Physical Review Letters 86(21), p. 4815-4818 (2001).

9. Leyrer et al, Mechano-Optical Octave-Tunable Elastic Colloidal Crystals Made from Core-Shell Polymer Beads with Self-Assembly Techniques, Langmuir 23, p 2961-2969 (2007). 


\title{
PROCEEDINGS OF SPIE
}

\section{Photonics: Design, Technology, and Packaging III}

\author{
Wieslaw Z. Krolikowski \\ Costas M. Soukoulis \\ Ping Koy Lam \\ Timothy J. Davis \\ Shanhui Fan \\ Yuri S. Kivshar \\ Editors
}

5-7 December 2007

Canberra, Australia

Sponsored by

SPIE

Cosponsored by

The Australian National University (Australia)

Australian Research Council Nanotechnology Network (Australia)

COSNet-ARC Complex Open Systems Research Network (Australia)

CUDOS (Australia)

RPO (Australia)

Bandwidth Foundry (Australia)

Cooperating Organizations

Research School of Physical Sciences and Engineering, Canberra (Australia)

Centre for Biomedical Engineering, Adelaide (Australia)

Published by

SPIE

Volume 6801 